\title{
Computing the critical points of the distance function between two Keplerian orbits via rigorous global optimization
}

\author{
R. Armellin - P. Di Lizia - M. Berz • K. Makino
}

Received: 27 November 2009 / Revised: 18 February 2010 / Accepted: 20 April 2010 /

Published online: 30 May 2010

(C) Springer Science+Business Media B.V. 2010

\begin{abstract}
A novel method to compute all critical points of the distance function between two Keplerian orbits (either bounded or unbounded) with a common focus is presented. The problem is attacked as a global optimization problem, solved by a rigorous global optimizer based on Taylor models. Thus, thigh enclosures of the stationary points are obtained. The embedded capability of the method of delivering high-order Taylor expansions is then used to analyze how uncertain orbital parameters affect the position of the stationary points and the associated distance values. Sample orbital sets and Apophis asteroid are used as test cases.
\end{abstract}

Keywords Taylor models · Rigorous computation - MOID · Asteroids and comets · Close encounters $\cdot$ Apophis

\section{Introduction}

The probability of a close encounter or an impact between two bodies moving on Keplerian orbits around the same attractor can be assessed by computing the minimum distance allowed by the geometry of the problem. In astrodynamics this quantity is usually referred to as minimum orbital intersection distance (MOID), and is usually computed by looking at all the stationary points of the square of the Euclidean distance, $d^{2}$, between two points on the first and the second orbit, respectively. The square is used to have a

R. Armellin $(\bowtie) \cdot$ P. Di Lizia

Dipartimento di Ingegneria Aerospaziale, Politecnico di Milano, Via La Masa 34, 20156 Milano, Italy e-mail: armellin@aero.polimi.it

P. Di Lizia

e-mail:dilizia@aero.polimi.it

M. Berz · K. Makino

Department of Physics and Astronomy, Michigan State University, East Lansing, MI 48824, USA e-mail: berz@msu.edu

K. Makino

e-mail: makino@msu.edu 
smooth function of the angular variables on the orbits also when the MOID is zero. Several algorithms have been proposed in the past (see for example Sitarski 1968, Hoots 1984, and Dybczynski et al. 1986) for the solution of this problem, which were mainly affected by the difficulty in dealing with a nonlinear one-dimensional equation appearing when a component of the critical points of $d^{2}$ is sought for. In Kholshevnikov and Vassiliev (1999) the problem was algebraically solved in the case of two Keplerian elliptic orbits by finding all the critical points of a trigonometric polynomial of degree 8, obtained with Gröbner bases theory. Furthermore, it was proven that a trigonometric polynomial of degree less than 8 does not exist. Later in Baluyev and Kholshevnikov (2005) the method was extended to all types of conic sections. In Gronchi (2002) an algorithm based on the resultant theory and the Fast Fourier Transform (FFT) is introduced to perform the elimination of one variable; an upper bound on the maximum number of critical points (if they are finitely many) is also obtained by using Newton's polytopes and Bernstein's theorem. Several improvements to the algorithm were later presented in Gronchi (2005) as well as the extension to unbounded Keplerian orbits.

In this paper the computation of MOID is approached as a global optimization problem. The rigorous global optimizer COSY-GO (Makino and Berz 2005) is run on either the square distance function or the square of its gradient for the computation of the MOID or all the stationary points of $d^{2}$, respectively. The advantages of using COSY-GO are numerous:

1. The optimizer is a branch-and-bound algorithm based on Taylor models (Makino 1998). As Taylor models compute validated enclosures of sufficiently smooth functions, the result is a tight rigorous enclosure of the global minimum of the objective function.

2. The problem formulation requires the analytical computation of $d^{2}$ and its first and second derivatives, which is rather simple.

3. When representing the objective function by a Taylor model, we also obtain the local slope, the Hessian and higher order derivatives almost for free. As a result, stationary points classification is straightforward.

The computation of the MOID of real celestial bodies, e.g. for the determination of potentially hazardous asteroids (PHA) or for debris analysis, requires some additional considerations. Firstly, the orbit parameters of the bodies are affected by uncertainty produced by the errors in the observations, which are usually represented by a covariance matrix (Milani 1999). The errors in the orbit determination also affect the computation of the MOID, and it is important to estimate the resulting uncertainty. Secondly, in a real scenario where many gravitational and non-gravitational perturbations are present, the Keplerian motion is an approximation, as the orbital parameters vary with time. In Gronchi et al. (2007) a regularization of the distance map is proposed and the covariance of the regularized maps is computed for a large database of orbits, with the goal of looking at PHA. In this work we propose three algorithms based on high-order Taylor expansions of the square distance function around the stationary points to address the problem of uncertain orbital parameters. In particular we present:

1. an algorithm that computes the range of the distance function at the stationary points when orbital parameters are given as intervals;

2. a fast and accurate Monte Carlo simulation for the fully nonlinear propagation of the covariance matrix, when the statistics of orbital parameters are available;

3. an algorithm that rigorously identifies, within the uncertainty set, the values of the orbital parameters that minimize the MOID.

The paper is organized as follows. Section 2 contains the problem formulation. In Sects. 3 and 4 some notes on the theory of Taylor models are given and the rigorous global optimizer COSY-GO is briefly described. A simple algorithm for the computer-assisted proof of the 
existence of stationary points is introduced in Sect. 5, and its application to several test cases is described in Sect. 6. Sections 7 and 8 are devoted to the explanation of the algorithms for the management of sets of uncertain orbital parameters and to the discussion of the numerical experiments. Some final remarks conclude the paper.

\section{Critical points of the square function}

The problem formulation is based on the cometary elements $\left(r_{p}, e, i, \Omega, \omega, \vartheta\right)$ that are the perihelion distance, eccentricity, inclination, longitude of ascending node, perihelion argument, and true anomaly, respectively. The geometrical configuration of the orbit is defined by the subset of the first 5 values (constants of motion), whereas the true anomaly is a parameter along the trajectory. In the reminder of the paper we will consider two orbits, labeled by either the subscripts 1 and 2 or $k$. Each orbit is parametrized on its orbit plane by

$$
\left\{\begin{array}{c}
\tilde{x}_{k}=r_{k} \cos \vartheta_{k} \\
\tilde{y}_{k}=r_{k} \sin \vartheta_{k}
\end{array}\right.
$$

where $r_{k}=l_{k} /\left(1+e_{k} \cos \vartheta_{k}\right)$ is the focal distance and $l_{k}=r_{p_{k}}\left(1+e_{k}\right)$ is the semi-latus rectum. As shown in Gronchi (2005), we can write the components of the orbits $\boldsymbol{x}_{k}=$ $\left(x_{k}, y_{k}, z_{k}\right)$ as

$$
\boldsymbol{x}_{k}=\tilde{x}_{k} \boldsymbol{p}_{k}+\tilde{y}_{k} \boldsymbol{q}_{k}
$$

in which the components of the vectors

$$
\begin{aligned}
& \boldsymbol{p}_{k}=\left(p_{x_{k}}, p_{y_{k}}, p_{z k}\right)^{T} \\
& \boldsymbol{q}_{k}=\left(q_{x_{k}}, q_{y_{k}}, q_{z k}\right)^{T}
\end{aligned}
$$

are defined by

$$
\begin{aligned}
& p_{x_{1}}=\cos \omega_{1} ; \quad p_{y_{1}}=\sin \omega_{1} \cos i_{1} ; \quad p_{z 1}=\sin \omega_{1} \sin i_{1} ; \\
& q_{x_{1}}=-\sin \omega_{1} ; \quad q_{y_{1}}=\cos \omega_{1} \cos i_{1} ; \quad q_{z 1}=\cos \omega_{1} \sin i_{1} ; \\
& p_{x_{2}}=\cos \omega_{2} \cos \left(\Omega_{2}-\Omega_{1}\right)-\sin \omega_{2} \cos i_{2} \sin \left(\Omega_{2}-\Omega_{1}\right) ; \\
& p_{y_{2}}=\cos \omega_{2} \sin \left(\Omega_{2}-\Omega_{1}\right)+\sin \omega_{2} \cos i_{2} \cos \left(\Omega_{2}-\Omega_{1}\right) ; \\
& p_{z 2}=\sin \omega_{2} \sin i_{2} ; \\
& q_{x_{2}}=-\sin \omega_{2} \cos \left(\Omega_{2}-\Omega_{1}\right)-\cos \omega_{2} \cos i_{2} \sin \left(\Omega_{2}-\Omega_{1}\right) ; \\
& q_{y_{2}}=-\sin \omega_{2} \sin \left(\Omega_{2}-\Omega_{1}\right)+\cos \omega_{2} \cos i_{2} \cos \left(\Omega_{2}-\Omega_{1}\right) ; \\
& q_{z 2}=\cos \omega_{2} \sin i_{2} .
\end{aligned}
$$

The distance function between two points belonging to two assigned Keplerian orbits is function of the true anomalies only, and its square can be computed by

$$
d^{2}\left(\vartheta_{1}, \vartheta_{2}\right)=\left(\boldsymbol{x}_{1}-\boldsymbol{x}_{2}\right)^{T} \cdot\left(\boldsymbol{x}_{1}-\boldsymbol{x}_{2}\right)
$$

The components of $\nabla d^{2}$ are

$$
\left\{\begin{array}{l}
\nabla_{\vartheta_{1}} d^{2}=\frac{r_{1}}{l_{1}}\left[e_{1} r_{1} \tilde{y}_{1}+\tilde{y}_{1}\left(K \tilde{x}_{2}+M \tilde{y}_{2}\right)-\left(e_{1} r_{1}+\tilde{x}_{1}\right)\left(L \tilde{x}_{2}+N \tilde{y}_{2}\right)\right] \\
\nabla_{\vartheta_{2}} d^{2}=\frac{r_{2}}{l_{2}}\left[e_{2} r_{2} \tilde{y}_{2}+\tilde{y}_{2}\left(K \tilde{x}_{2}+L \tilde{y}_{1}\right)-\left(e_{2} r_{2}+\tilde{x}_{2}\right)\left(M \tilde{x}_{1}+N \tilde{y}_{1}\right)\right]
\end{array}\right.
$$


where

$$
K=\boldsymbol{p}_{1}^{T} \cdot \boldsymbol{p}_{2} ; \quad L=\boldsymbol{q}_{1}^{T} \cdot \boldsymbol{p}_{2} ; \quad M=\boldsymbol{p}_{1}^{T} \cdot \boldsymbol{q}_{2} ; \quad N=\boldsymbol{q}_{1}^{T} \cdot \boldsymbol{q}_{2} .
$$

The problem of computing both the MOID and the stationary points (SP) of $d^{2}$ can be approached as a rigorous global optimization problem by defining the two objective functions

$$
\mathcal{J}_{\operatorname{MOID}}\left(\vartheta_{1}, \vartheta_{2}\right)=d^{2}
$$

and

$$
\mathcal{J}_{\mathrm{SP}}\left(\vartheta_{1}, \vartheta_{2}\right)=\nabla d^{2^{T}} \cdot \nabla d^{2}
$$

respectively. As finding the zeros of the components of (4) is equivalent to finding the zeros of the two nonlinear functions (see Gronchi 2005)

$$
\left\{\begin{aligned}
f_{1}\left(\vartheta_{1}, \vartheta_{2}\right)= & l_{2}\left(1+e_{1} \cos \vartheta_{1}\right)\left[\sin \vartheta_{1}\left(K \cos \vartheta_{2}+M \sin \vartheta_{2}\right)\right. \\
& \left.-\left(e_{1}+\cos \vartheta_{1}\right)\left(L \cos \vartheta_{2}+N \sin \vartheta_{2}\right)\right]+e_{1} l_{1} \sin \vartheta_{1}\left(1+e_{2} \cos \vartheta_{2}\right) \\
f_{2}\left(\vartheta_{1}, \vartheta_{2}\right)= & l_{1}\left(1+e_{2} \cos \vartheta_{2}\right)\left[\sin \vartheta_{2}\left(K \cos \vartheta_{1}+L \sin \vartheta_{1}\right)\right. \\
& \left.-\left(e_{2}+\cos \vartheta_{2}\right)\left(M \cos \vartheta_{1}+N \sin \vartheta_{1}\right)\right]+e_{2} l_{2} \sin \vartheta_{2}\left(1+e_{1} \cos \vartheta_{1}\right)
\end{aligned}\right.
$$

the objective function (6) can be replaced by

$$
\mathcal{J}_{\mathrm{SP}}\left(\vartheta_{1}, \vartheta_{2}\right)=f_{1}^{2}+f_{2}^{2} .
$$

The search space is defined by $1+e_{k} \cos \vartheta_{k}>0$; for bounded orbits this is verified when $-\pi \leq \vartheta_{k}<\pi$, whereas for unbounded orbits when $-\cos ^{-1}\left(-1 / e_{k}\right)<\vartheta<\cos ^{-1}\left(-1 / e_{k}\right)$. To reduce the number of parameters involved in the computation of the objective function it is convenient to adopt the cometary mutual elements introduced in Gronchi (2005). In particular, the transformation from cometary elements to mutual cometary elements is

$$
\begin{aligned}
& \mathcal{E}_{1}=\left(r_{p_{1}}, e_{1}, i_{1}, \Omega_{1}, \omega_{1}\right)=\left(r_{p_{1}}, e_{1}, 0,0, \omega_{1}^{M}\right), \\
& \mathcal{E}_{2}=\left(r_{p_{2}}, e_{2}, i_{2}, \Omega_{2}, \omega_{2}\right)=\left(r_{p_{2}}, e_{2}, i^{M}, 0, \omega_{2}^{M}\right),
\end{aligned}
$$

in which $i^{M}$ is the mutual inclination between the orbital planes, and $\omega_{1}^{M}, \omega_{2}^{M}$ are the angles between the ascending mutual node of the second orbit with respect to the first orbit and the pericenters of the two orbits. Note that the map from ordinary cometary elements to the mutual elements is not injective as there are infinitely many configurations that brings to the same mutual position of the two orbits.

\section{Notes on Taylor models}

Verified global optimization needs the determination of rigorous upper and lower bounds of the objective function in order to implement a branch-and-bound method (Kearfott 1996). The commonly used interval approach has excelled in solving this problem elegantly from both a formal and an implementational viewpoint. However, there are situations where the method has limitations for extended or complicated calculations because of the dependency problem, which is characterized by a cancellation of various sub-parts of the function that cannot be detected by direct use of interval methods. This effect often leads to pessimism and sometimes even drastic overestimation of range enclosure. Furthermore, the sharpness of 
intervals resulting from calculations typically scales linearly with the sharpness of the initial discretization intervals. For complicated problems, and in particular higher dimensions, this sometimes significantly limits the sharpness of the resulting answer that can be obtained (Makino and Berz 1999).

The Taylor model approach enables the computation of fully mathematically rigorous range enclosures while largely avoiding many of the limitations of the conventional interval method (Makino 1998). The method is based on the inductive local modelling of functional dependencies by a polynomial with a rigorous remainder bound, and as such represents a hybrid between formula manipulation, interval methods, and methods of computational differentiation (Berz et al. 1996; Griewank and Corliss 1991).

An $n$-th order Taylor model of a multivariate function $f$ that is $(n+1)$ times continuously partially differentiable on the domain $D$, consists of the $n$-th order multivariate Taylor polynomial $P$ expanded around a point $x_{0} \in D$ and representing a high-order approximation of the function $f$, and a remainder error interval $I$ for verification such that

$$
\forall x \in D, \quad f(x) \in P\left(x-x_{0}\right)+I .
$$

From Taylor's theorem, it is clear that the width of the remainder interval $I$ can be chosen to scale with the domain size proportional to $\left|\boldsymbol{x}-\boldsymbol{x}_{0}\right|^{n+1}$. The practical computation of $P$ and $I$ is based on Taylor model arithmetic, which carries $P$ and $I$ through all the operations comprising $I$. By choosing the size $\left|\boldsymbol{x}-\boldsymbol{x}_{0}\right|$ sufficiently small and the order $n$ sufficiently high, the size of the remainder interval $I$ can be kept very small in practice. The bulk of the functional dependency is kept in the polynomial part $P$ with point coefficients, and there is no interval arithmetic associated inflation that happens in the polynomial part. Thus, the interval related overestimation is rather optimally suppressed with the Taylor model method (Makino and Berz 1999). The implementation of the method in the code COSY Infinity (Berz and Makino 2006; Makino 1998), supports binary operations and standard intrinsic functions, as well as the antiderivative operation which widens the applications of the method. Note that when only the polynomial part $P$ of the Taylor model is considered, also the analytic operation of differentiation can be introduced, so finalizing the definition of a differential algebraic (DA) structure (Berz 1999).

The Taylor model approach has the following important properties:

1. The ability to provide rigorous enclosures of any function given by a finite computer code list by a Taylor polynomial and a remainder bound with a sharpness that scales with order $(n+1)$ of the width of the domain.

2. The computational expense increases only moderately with order, allowing the computation of sharp range enclosures even for complicated functional dependencies with significant dependency problem.

3. The computational expense of higher dimensions increases only very moderately, significantly reducing the "curse of dimensionality".

The structure of Taylor models naturally represents a rich resource of information. In particular, the coefficients of the polynomial part $P$ of a Taylor model are nothing but the derivatives up to order $n$. Consequently, when representing a function $f$ by a Taylor model $P+I$ on a computer, we also obtain the local slope, Hessian and higher order derivatives. When a task is focused on range bounding, those pieces of information become particularly useful.

While naive range bounding of Taylor models, namely merely evaluating each monomial of $P$ using interval arithmetic then summing up all the contributions as well as the remainder interval I (Makino and Berz 2003), already exhibits the superiority over the mere interval 
arithmetic and the more advanced centered form (Makino 1998), the active utilization of those additional pieces of information in Taylor models has a lot of potential of developing efficient range bounders. Based on this observation, various kinds of Taylor model based range bounders have been developed (Berz et al. 2005), and among them the linear dominated bounder (LDB) and the quadratic fast bounder (QFB) are the backbones of Taylor model based verified global optimizer COSY-GO that will be discussed afterward.

The linear dominated bounder is based on the fact that for Taylor models with sufficiently small remainder bound, the linear part of the Taylor model dominates the behavior, and this is also the case for range bounding. The linear dominated bounder utilizes the linear part as a guideline for iterative domain reduction to bound Taylor models. Around an isolated interior minimizer, the Hessian of a function $f$ is positive definite, so the purely quadratic part of a Taylor model $(P, I)$ which locally represents $f$, has a positive definite Hessian matrix $H$. The quadratic fast bounder provides a lower bound of a Taylor model cheaply when the purely quadratic part is positive definite. More details on polynomial bounders are given in Makino and Berz (2005).

\section{COSY-GO}

COSY-GO (Berz et al. 2005) is a branch-and-bound optimization algorithm employing local domain reduction techniques exploiting the bounding performances assured by Taylor model methods. Should the global minimum of a sufficiently regular scalar function $f$ on a given domain $A \subseteq \mathbb{R}^{m}$ wished to be evaluated, the algorithm starts with an initial value for the global optimum, the cutoff value, and then proceeds on analyzing at each step a subdomain for possible elimination or reduction. At each step the following tasks are performed:

1. A rigorous lower bound $l$ of the objective function is obtained on the subdomain of interest using various bounding schemes hierarchically with the hope of showing that $l$ lies above the already established cutoff value, which will allow elimination of the subdomain. A first assessment is made whether the remainder bound of the Taylor model at hand is sufficiently small; if it is not, then the underlying function exhibits too much detail for modeling by local estimators, and the subdomain is split in the direction of fastest change of the function.

2. If the remainder bound is sufficiently small, as a first test the polynomial part of the objective function is evaluated in interval arithmetic. When it fails to eliminate the box, the LDB bounder is applied. If it also fails to eliminate the box, and if the quadratic part of the polynomial representation of the objective function $P$ is positive definite, the QFB bounder is applied.

3. If the just studied subdomain of interest cannot be eliminated, but is seen to have a lower bound close to the current cutoff values, domain reduction techniques are brought to bear based on the LDB and QFB algorithms to reduce the subdomain in size. Once these methods are applicable, they will allow to cut the subdomain of interest and rapidly reduce the active volume.

4. The cutoff value is updated using various schemes. First, the linear and quadratic parts of the Taylor polynomial are utilized to obtain a potential cutoff update. In particular, if the quadratic part of the polynomial is positive definite, the minimizer of the quadratic polynomial is tested. If the quadratic part is not positive definite, the minimizer of the quadratic part in the direction of the negative gradient is tested. For objective functions of 
nontrivial cost, as in the example at hand, also more sophisticated local searches within and near the current subdomain may be carried out.

The algorithm continues to reduce and examine the domain until the minimum dimension allowed is reached. The result of the optimization is the validated enclosure of the global minimum of the problem.

\section{Local proof of the existence and uniqueness of stationary points}

In Sect. 2 we have formulated the problem of both MOID and stationary points computation as an optimization problem, being $d^{2}$ and $f_{1}^{2}+f_{2}^{2}$ the objective functions. By running COSY-GO on $d^{2}$, the validated enclosure of the MOID is obtained with a width that depends on the selected expansion order and minimum dimension allowed for the domain. In the same way, when applied to $f_{1}^{2}+f_{2}^{2}$, COSY-GO delivers the validated enclosure of the minima of this function: a set of $m$ interval vectors $\boldsymbol{\Theta}_{i}=\left[\underline{\vartheta}_{i}, \bar{\vartheta}_{i}\right] \subset \mathbb{S}$ with $i=1, \ldots, m$, that are only candidate enclosures of the stationary points of $d^{2}$. (The set $\mathbb{S}$ is a torus in case of two bounded conics, an open cylinder in case of one bounded and one unbounded conic, and an open square in case of two unbounded conics.)

We need to prove that each interval $\Theta_{i}$ contains a unique solution of the nonlinear system

$$
\boldsymbol{f}(\boldsymbol{\vartheta})=\mathbf{0},
$$

in which $\boldsymbol{f}(\vartheta)=\left(f_{1}\left(\vartheta_{1}, \vartheta_{2}\right), f_{2}\left(\vartheta_{1}, \vartheta_{2}\right)\right) \in \mathcal{C}^{\infty}(\mathbb{S})$. To this aim we consider the function $\boldsymbol{g}(\boldsymbol{\vartheta})$ defined as

$$
\boldsymbol{g}(\boldsymbol{\vartheta})=\vartheta-Q \cdot \boldsymbol{f}(\boldsymbol{\vartheta}),
$$

where $Q$ is the inverse of the Jacobian of $\boldsymbol{f}$ evaluated at the midpoint of $\boldsymbol{\Theta}_{i}$, i.e. $Q=$ $\left[J_{f}\left(\boldsymbol{\vartheta}_{m p}\right)\right]^{-1}$. Note that $\boldsymbol{g}$ is continuously differentiable on the convex and compact set $\boldsymbol{\Theta}_{i}$. From Banach's fixed point theorem, if $\boldsymbol{g}$ is a contraction on $\boldsymbol{\Theta}_{i}$ then a unique point $\boldsymbol{\vartheta}^{*} \in \boldsymbol{\Theta}_{i}$ such that $\boldsymbol{g}\left(\vartheta^{*}\right)=\vartheta^{*}$ exists. By construction, from Eq. 11 it also follows that $\vartheta^{*}$ is the unique solution of $\boldsymbol{f}(\boldsymbol{\vartheta})=\mathbf{0}$ in $\boldsymbol{\Theta}_{i}$.

In order to prove the existence of a unique fixed point we use Taylor models algebra to show that

$$
\left|\lambda_{J_{g}, k}(\vartheta)\right|<1 \forall \vartheta \in \boldsymbol{\Theta}_{i}, \text { with } k=1,2 ;
$$

where $\lambda_{J_{g}, k}$ are the eigenvalues of the Jacobian of $\boldsymbol{g}$ over $\boldsymbol{\Theta}_{i}$. The Jacobian of $\boldsymbol{g}$ is given by

$$
J_{g}(\vartheta)=\mathcal{I}-Q \cdot J_{f}(\vartheta),
$$

where $\mathcal{I}$ is the identity matrix and the Jacobian $J_{\boldsymbol{f}}(\boldsymbol{\vartheta})$ is computed analytically. Map (13) can be rigorously evaluated in the Taylor model framework obtaining

$$
J_{\boldsymbol{g}}(\boldsymbol{\vartheta})=\mathcal{I}-Q \cdot J_{f}(\boldsymbol{\vartheta}) \in \boldsymbol{P}(\boldsymbol{\vartheta})+I, \quad \forall \vartheta \in \boldsymbol{\Theta}_{i}
$$

The fulfillment of condition (12) can be rigorously checked by computing the Taylor models of the determinant and the trace of $J_{g}(\vartheta)$ and by bounding them over the domain $\boldsymbol{\Theta}_{i}$ by means of the rigorous polynomial bounder LDB. If both the trace and determinant are in the set $(-1 / 2,1 / 2)$, then condition (12) is satisfied. Checking the range of the determinant and the trace has the advantage of avoiding the numerical issues that arise in the validated computation of the eigenvalues when their range is small and close to zero over the domain of interest. 
Table 1 Test cases definition

\begin{tabular}{llllllrr}
\hline Test case \# & $r_{p_{1}}$ & $e_{1}$ & $r_{p_{2}}$ & $e_{2}$ & $i^{M}$ & $w_{1}^{M}$ & $w_{2}^{M}$ \\
\hline & $\mathrm{AU}$ & - & $\mathrm{AU}$ & \multicolumn{1}{c}{-} & $\mathrm{deg}$ & $\mathrm{deg}$ & $\operatorname{deg}$ \\
\hline 1 & 1.0 & 0.0 & 0.48 & 0.6 & 60.0 & 16.0 & 176.0 \\
2 & 0.585 & 0.415 & 0.462 & 0.615 & 80.0 & 8.0 & 176.0 \\
3 & 1.0 & 0.6 & 1.2 & 1.1 & 40.0 & 73.0 & 69.0 \\
4 & 1.0 & 0.5 & 1.2 & 1.1 & 66.0 & 4.0 & 136.0 \\
\hline
\end{tabular}

Table 2 Apophis equinoctial variables at $3456 \mathrm{MJD} 2000$ (June $18,2009)$ and associated $\sigma$ values

\begin{tabular}{lrll}
\hline & \multicolumn{1}{l}{ Nom value } & \multicolumn{1}{l}{$\sigma$} \\
\hline$a$ & 0.922438242375914 & $2.29775 \times 10^{-8}$ & AU \\
$P_{1}$ & -0.093144699837425 & $3.26033 \times 10^{-8}$ & - \\
$P_{2}$ & 0.166982492089134 & $7.05132 \times 10^{-8}$ & - \\
$Q_{1}$ & -0.012032857685451 & $5.39528 \times 10^{-8}$ & - \\
$Q_{2}$ & -0.026474053361345 & $1.83533 \times 10^{-8}$ & - \\
$l$ & 88.3150906433494 & $6.39035 \times 10^{-5}$ & deg \\
\hline
\end{tabular}

Once map (11) is proven to be a contraction on $\boldsymbol{\Theta}_{i}$, a finer enclosure of the fixed point $\boldsymbol{\vartheta}^{*}$ can be obtained by iterative evaluations of map (11).

\section{Critical points of the square function: Numerical experiments}

In this section we present the results obtained by running COSY-GO on the problem formulated in Sect. 2 using some of the sets of orbital parameters analyzed in Gronchi (2005) (see Table 1) and asteroid Apophis (see Table 2) as test cases. The orbital parameters of Apophis are computed from the equinoctial variables available at the Near Earth Objects Dynamic Site (newton.dm.unipi.it/neodys) for the date June 18, 2009; at the same date, JPL planetary ephemerides DE405 (http://ssd.jpl.nasa.gov/?horizons) are used for the Earth. The transformation from equinoctial variables to cometary elements is

$$
\begin{aligned}
e & =\sqrt{P_{1}^{2}+P_{2}^{2}} & & \tan \frac{1}{2} i=\sqrt{Q_{1}^{2}+Q_{2}^{2}} \\
\tan (\omega+\Omega) & =P_{1} / P_{2} & & \tan \Omega=Q_{1} / Q_{2}
\end{aligned}
$$

together with $r_{p}=a(1-e)$ (Battin 1987).

All the simulations are performed on a Mac Mini $2 \mathrm{GHz}$ Intel Core 2 Duo, running Mac OS X Version 10.5.7. The minimum dimension allowed for both the variables defining the search space is $1 \times 10^{-6} \mathrm{deg}$ and the expansion order is set to 6 , as at higher orders most of the coefficients of the Taylor expansion become too small and their contribution is moved to the remainder interval.

Figure $1 \mathrm{a}$ and $1 \mathrm{~b}$ are relative to the first test case. The landscape of the two objective functions (5) and (8) are represented through color maps and contour lines. The black lines superimposed to this representation show the optimizer's box splitting, reduction and elimination sequence. Table 3 lists the enclosures of all the stationary points of the function of Fig. 1a, the corresponding enclosures of the distance function $d$, and the type of singularity. 

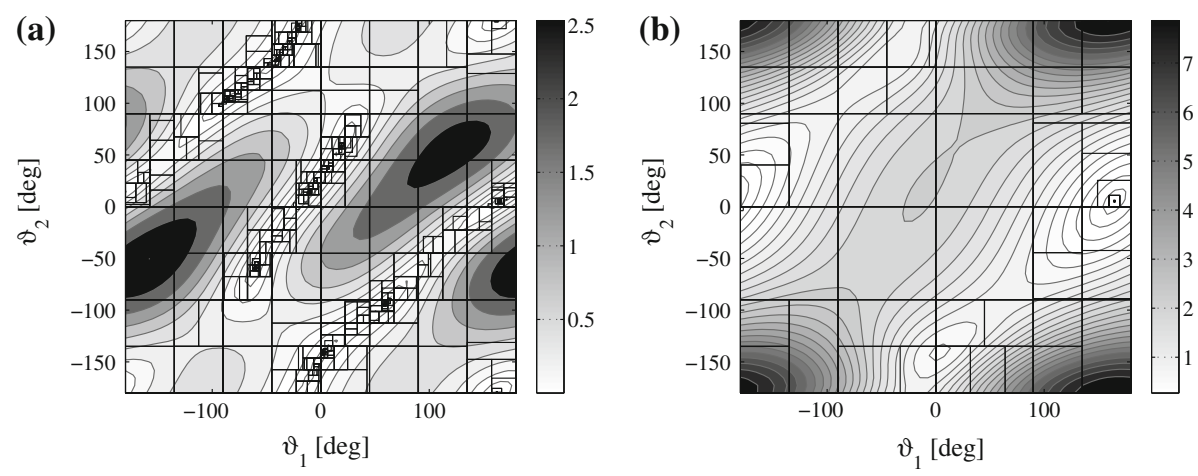

Fig. 1 Stationary points and MOID computation for the first test case. a Objective function landscape and COSY-GO box splitting, reduction and elimination sequence for the computation of stationary points. b Objective function landscape and COSY-GO box splitting, reduction and elimination sequence for MOID computation

Table 3 Enclosure of stationary points for the first test case

\begin{tabular}{llll}
\hline$\vartheta_{1}$ & $\vartheta_{2}$ & $d$ & Type \\
\hline $\operatorname{deg}$ & deg & AU & - \\
\hline$[164.701274,164.701280]$ & {$[5.402343,5.402345]$} & {$[0.519406,0.519408]$} & MOID \\
{$[18.443020,18.443022]$} & {$[57.905829,57.905832]$} & {$[1.473478,1.473480]$} & Saddle \\
{$[162.290768,162.290774]$} & {$[-179.415425,-179.415419]$} & {$[2.918973,2.918975]$} & Maximum \\
{$[60.526169,60.526171]$} & {$[-92.831357,-92.831355]$} & {$[0.904612,0.904614]$} & Saddle \\
{$[3.187965,3.187967]$} & {$[-141.161981,-141.161975]$} & {$[0.756873,0.756875]$} & Minimum \\
{$[-20.410603,-20.410601]$} & {$[175.230455,175.230461]$} & {$[0.928271,0.928273]$} & Saddle \\
{$[-39.540706,-39.540703]$} & {$[142.933879,142.933885]$} & {$[0.864582,0.864584]$} & Minimum \\
{$[-85.283891,-85.283889]$} & {$[104.707906,104.707912]$} & {$[0.932249,0.932251]$} & Saddle \\
{$[-10.066183,-10.066180]$} & {$[15.743011,15.743013]$} & {$[1.481712,1.481714]$} & Maximum \\
{$[-60.116751,-60.116748]$} & {$[-58.721733,-58.721730]$} & {$[1.445876,1.445878]$} & Saddle \\
\hline
\end{tabular}

Note that the enclosures obtained in the simulations are much tighter than those reported in the tables throughout the paper; the number of significant digits has been reduced for visualization aims. Ten stationary points are identified for the first test case: three minima (the global one being the MOID), five saddle points, and two maxima. As we use Taylor models, the classifications of these points is straightforwardly performed by examining the quadratic part of the Taylor expansion of the distance function on the boxes remaining after the optimization process. The overall process for the stationary points computation, check of uniqueness, and classification takes $0.88 \mathrm{~s}$ as indicated in Table 4, which summarizes the CPU times for all the test cases.

It is illuminating to observe the details of box reduction close to the global optimum in Fig. 1b. As in this region the Hessian of the objective function is positive definite, the QFB algorithm is used to perform a sequence of efficient box reductions. Furthermore, the high accuracy of the Taylor model representation of the objective function avoids significant box splitting and box reduction close to the two local minima. In this case the computational time 
Table 4 CPU time in seconds required for the computation of the stationary points and MOID for the different test cases and expansion order $n$ equal to 6 and 2

\begin{tabular}{lllllll}
\hline & & $\# 1$ & $\# 2$ & $\# 3$ & $\# 4$ & Apophis \\
\hline Stationary points & $n=6$ & 0.88 & 0.72 & 0.95 & 1.45 & 2.39 \\
& $n=2$ & 0.41 & 0.34 & 0.44 & 0.61 & 1.00 \\
MOID & $n=6$ & 0.14 & 0.24 & 0.16 & 0.26 & 0.18 \\
& $n=2$ & 0.05 & 0.09 & 0.08 & 0.12 & 0.09 \\
\hline
\end{tabular}
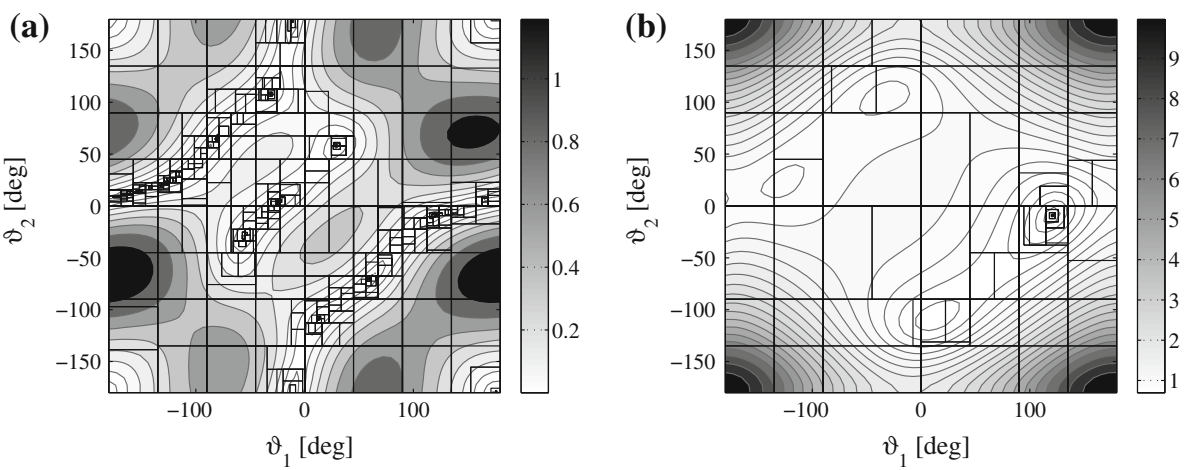

Fig. 2 Stationary points and MOID computation for the second test case. a Objective function landscape and COSY-GO box splitting, reduction and elimination sequence for the computation of stationary points. b Objective function landscape and COSY-GO box splitting, reduction and elimination sequence for MOID computation
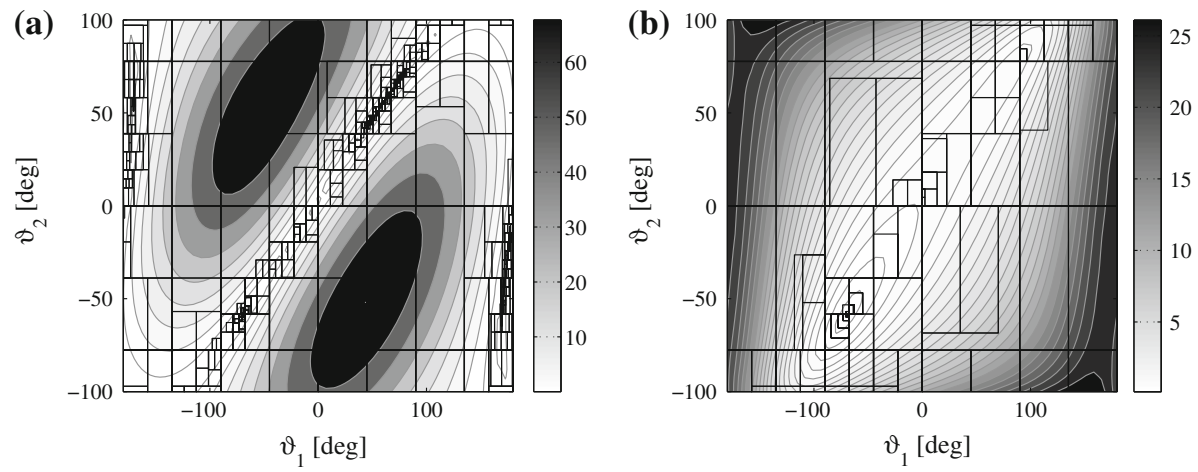

Fig. 3 Stationary points and MOID computation for the third test case. a Objective function landscape and COSY-GO box splitting, reduction and elimination sequence for the computation of stationary points. b Objective function landscape and COSY-GO box splitting, reduction and elimination sequence for MOID computation

lowers to $0.14 \mathrm{~s}$. When the computation of MOID is of interest, it is convenient to run the global optimizer on the square of the distance function only.

Figures 2, 3 and 4 and Tables 5, 6 and 7 summarize the results for test cases 2-4. The second test case considers two bounded orbits with 12 stationary points. In both the third and the fourth examples an ellipse and a hyperbola are considered, resulting in the computation 

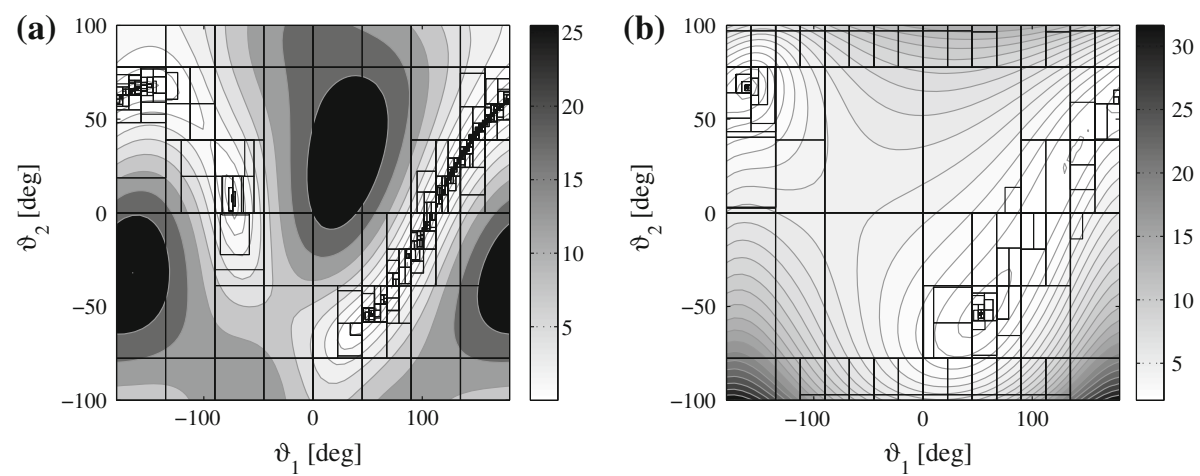

Fig. 4 Stationary points and MOID computation for the fourth test case. a Objective function landscape and COSY-GO box splitting, reduction and elimination sequence for the computation of stationary points. b Objective function landscape and COSY-GO box splitting, reduction and elimination sequence for MOID computation

Table 5 Enclosure of stationary points for the second test case

\begin{tabular}{llll}
\hline$\vartheta_{1}$ & $\vartheta_{2}$ & $d$ & Type \\
\hline $\operatorname{deg}$ & deg & AU & - \\
\hline$[29.329042,29.329045]$ & {$[58.135699,58.135701]$} & {$[1.031590,1.031592]$} & Saddle \\
{$[120.685566,120.685570]$} & {$[-9.332882,-9.332880]$} & {$[0.833578,0.833580]$} & MOID \\
{$[176.166458,176.166462]$} & {$[-179.014041,-179.014037]$} & {$[3.346468,3.346468]$} & Maximum \\
{$[59.693876,59.693879]$} & {$[-70.405952,-70.405950]$} & {$[0.898021,0.898023]$} & Saddle \\
{$[12.711961,12.711964]$} & {$[-108.567127,-108.567123]$} & {$[0.868070,0.868072]$} & Minimum \\
{$[-11.199719,-11.199717]$} & {$[178.714334,178.714338]$} & {$[1.353077,1.353079]$} & Saddle \\
{$[-31.447002,-31.446999]$} & {$[107.562345,107.562349]$} & {$[0.947004,0.947006]$} & Minimum \\
{$[-24.517615,-24.517612]$} & {$[3.349973,3.349975]$} & {$[1.052484,1.052486]$} & Maximum \\
{$[-80.560168,-80.560165]$} & {$[65.783503,65.783506]$} & {$[0.975559,0.975561]$} & Saddle \\
{$[-127.417508,-127.417504]$} & {$[22.521944,22.521946]$} & {$[0.954157,0.954159]$} & Minimum \\
{$[-164.745176,-164.745172]$} & {$[10.898725,10.898727]$} & {$[0.969579,0.969581]$} & Saddle \\
{$[-54.548772,-54.548770]$} & {$[-27.883057,-27.883054]$} & {$[1.048037,1.048039]$} & Saddle \\
\hline
\end{tabular}

of 6 stationary points. Note that for test case 4 the computation of MOID is quite challenging, as the three minima are characterized by very close values of the distance function.

The last test case considers the osculating orbital elements of asteroid Apophis and the Earth. This test case is of particular interest as Apophis will have a close encounter with the Earth on April 13, 2029. The possibility of this close encounter is highlighted by the very low value of the MOID reported in Table 8, which is in agreement with the value that can be found at http://newton.dm.unipi.it/neodys/index.php?pc=1.1. $0 \& n=99942$. As previously mentioned, the results displayed in the tables are rounded for visualization purposes: the actual enclosure of the MOID computed by COSY-GO is $[0.1730625171732279,0.1730625171888673] \times 10^{-3}$ AU. The CPU time required for the computation of the stationary points is quite high due to the presence of lines where the 
Table 6 Enclosure of stationary points for the third test case

\begin{tabular}{llll}
\hline$\vartheta_{1}$ & $\vartheta_{2}$ & $d$ & Type \\
\hline $\operatorname{deg}$ & $\operatorname{deg}$ & AU & - \\
\hline$[76.748885,76.748888]$ & {$[69.259356,69.259358]$} & {$[0.817428,0.817430]$} & Minimum \\
{$[46.838191,46.838194]$} & {$[44.616704,44.616707]$} & {$[0.833578,0.833580]$} & Saddle \\
{$[176.025979,176.025985]$} & {$[-20.460198,-20.460196]$} & {$[5.007250,5.007252]$} & Maximum \\
{$[169.888792,169.888798]$} & {$[-56.530124,-56.530121]$} & {$[5.000161,5.000163]$} & Saddle \\
{$[-169.888811,-169.888805]$} & {$[62.566044,62.566047]$} & {$[4.947316,4.947318]$} & Saddle \\
{$[-69.498772,-69.498770]$} & {$[-58.677054,-58.677051]$} & {$[0.346196,0.346198]$} & MOID \\
\hline
\end{tabular}

Table 7 Enclosure of stationary points for the fourth test case

\begin{tabular}{llll}
\hline$\vartheta_{1}$ & $\vartheta_{2}$ & $d$ & Type \\
\hline $\operatorname{deg}$ & $\operatorname{deg}$ & $\mathrm{AU}$ & - \\
\hline$[160.437997,160.438006]$ & {$[50.073804,50.073807]$} & {$[1.515413,1.515415]$} & Saddle \\
{$[138.661675,138.661681]$} & {$[32.795490,32.795493]$} & {$[1.508532,1.508534]$} & Minimum \\
{$[102.149380,102.149386]$} & {$[-8.352026,-8.352024]$} & {$[1.525643,1.525645]$} & Saddle \\
{$[52.859752,52.859755]$} & {$[-53.973031,-53.973029]$} & {$[1.487301,1.487303]$} & Minimum \\
{$[-73.558573,-73.558570]$} & {$[7.685115,7.685117]$} & {$[2.187974,2.187976]$} & Saddle \\
{$[-160.603625,-160.603619]$} & {$[66.664906,66.664908]$} & {$[1.442148,1.442150]$} & MOID \\
\hline
\end{tabular}

Table 8 Enclosure of stationary points for Apophis test case

\begin{tabular}{llll}
\hline$\vartheta_{1}$ & $\vartheta_{2}$ & $\mathrm{~d}$ & type \\
\hline $\mathrm{deg}$ & $\mathrm{deg}$ & $\mathrm{AU}$ & - \\
\hline$[41.007157,41.007159]$ & {$[174.506132,174.506138]$} & {$[0.121348,0.121350]$} & Saddle \\
{$[99.844426,99.844429]$} & $[-126.554012,-126.554006]]$ & {$[0.000172,0.000174]$} & MOID \\
{$[42.423025,42.423028]$} & {$[-5.472986,-5.472984]$} & {$[1.734156,1.734158]$} & Saddle \\
{$[-15.589186,-15.589183]$} & {$[118.047915,118.047921]$} & {$[0.052045,0.052047]$} & Minimum \\
{$[-137.139398,-137.139392]$} & {$[177.660138,177.660144]$} & {$[2.110186,2.110188]$} & Maximum \\
{$[-140.482353,-140.482347]$} & {$[-7.138379,-7.138377]$} & {$[0.269629,0.269631]$} & Saddle \\
\hline
\end{tabular}

function (8) assumes values close to zero, as visible from Fig. 5a. On the other hand, computing the MOID is faster thanks to the presence of only two minima (see Fig. 5b).

Table 4 shows that the computational time is strongly affected by the expansion order selected; the second order computations allows us to roughly half the CPU times. Furthermore, the fast polynomial bounders implemented in COSY-GO and the algorithm for domain reduction and elimination yield a computational time that is order of magnitudes lower than that required by verified global optimizers based on interval arithmetics. On the other hand, algorithms that exploit the reduction of the problem to the search of the solutions of a univariate polynomial equation, such as those proposed in Gronchi (2005) and Baluyev and Kholshevnikov (2005), are characterized by a better computational efficiency. The proposed method is alternative to them and allows us to obtain validated enclosures of the solutions. Moreover, the Taylor expansions available at the end of the optimization process enable the 

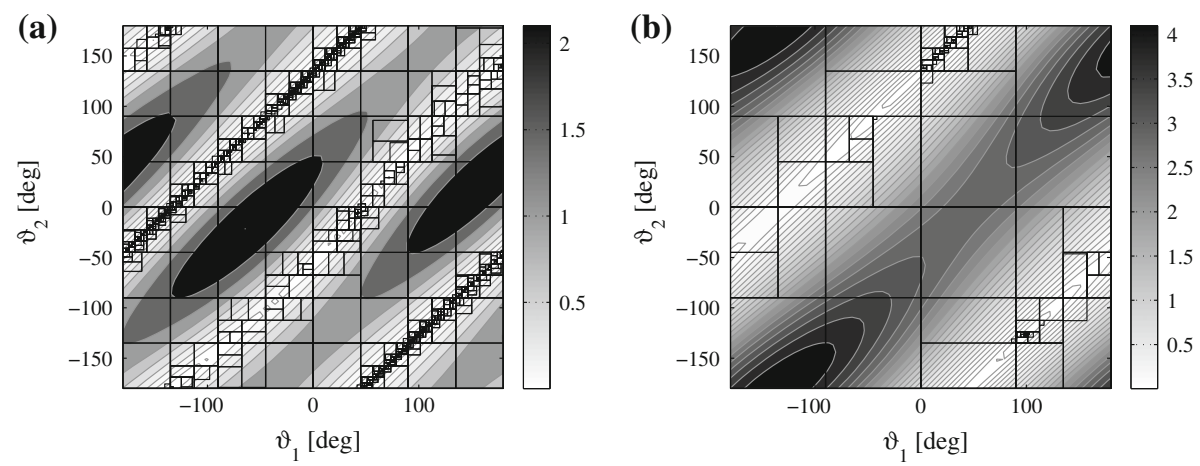

Fig. 5 Stationary points and MOID computation for Apophis test case. a Objective function landscape and COSY-GO box splitting, reduction and elimination sequence for the computation of stationary points. b Objective function landscape and COSY-GO box splitting, reduction and elimination sequence for MOID computation
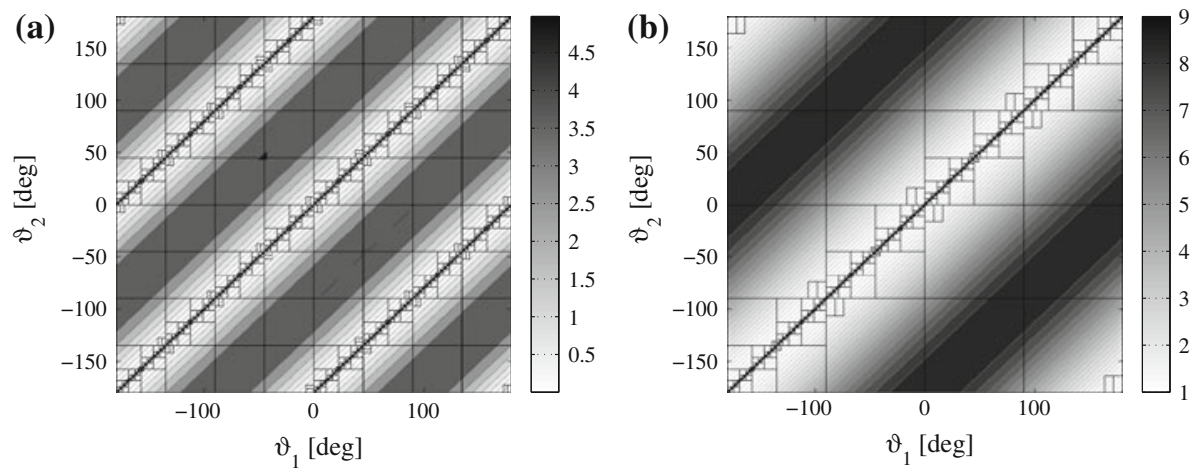

Fig. 6 Stationary points and MOID computation for the concentric coplanar circles case. a Objective function landscape and COSY-GO box splitting, reduction and elimination sequence for the computation of stationary points. b Objective function landscape and COSY-GO box splitting, reduction and elimination sequence for MOID computation

development of the algorithms to analyze the effects of uncertain orbital parameters on the stationary points and the associated distance values (see Sect. 7). As a final remark, note that Taylor models could be utilized to find all the solutions of the univariate polynomial equation obtained by the algebraic approaches, but this is left to future investigations.

The proposed approach works for most of the degenerate cases, even when infinitely many critical points occur, as for concentric coplanar circles or two overlapping conics. Figure 6 shows COSY-GO box splitting, reduction and elimination sequence when two concentric coplanar circles with $r_{p_{1}}=1$ and $r_{p_{2}}=2$ are considered. The result of the optimization process is the rigorous enclosure of the solution set $\vartheta_{1}=\vartheta_{2}$ and $\vartheta_{1}=\vartheta_{2} \pm \pi$ for the stationary points, and of $\vartheta_{1}=\vartheta_{2}$ for MOID. The CPU time required for computing these solutions is strongly dependent on the minimum dimension allowed for both the variables; this affects the number of required optimizer iterations (and thus the computational time) as well as the accuracy of the enclosure of the solution lines. With a minimum dimension of $0.1 \mathrm{deg}$ the optimization process takes $93.62 \mathrm{~s}$ for the computation of the enclosure of the 
stationary points and $43.23 \mathrm{~s}$ for MOID. Note that in this case each box left by the optimizer contains a line of stationary points, thus the algorithm of Sect. 5 is not applied.

The minimization method cannot manage the particular case when the non-compactness of $\mathbb{S}$ plays a role. As explained in Baluyev and Kholshevnikov (2005), this situation occurs when two hyperbolas (including rectilinear orbits as hyperbolic rays) with at least two parallel asymptotes are considered. In this case the MOID does not exist, and the set-theoretic distance between the orbits is the infimum of the distances between two points lying on the hyperbolas; i.e., the distance between the asymptotes.

\section{Uncertain orbital parameters}

The results shown in Sect. 6 considered pointwise sets of orbital parameters. When the computation of the MOID of real objects (e.g., for the determination of PHA or for debris applications) is of interest it is preferable to consider the orbital parameters as interval sets for the following reasons:

1. the orbital parameters of a real object are affected by uncertainties due to the orbital determination process;

2. in a real environment, the orbital parameters vary with time due to perturbations.

In order to address this problem, we introduce an algorithm that exploits Taylor differential algebra for expanding to high-orders the solution of the set of nonlinear equations

$$
\left\{\begin{array}{l}
l_{2}\left(1+e_{1} \cos \vartheta_{1}\right)\left[\sin \vartheta_{1}\left(K \cos \vartheta_{2}+M \sin \vartheta_{2}\right)\right. \\
\left.-\left(e_{1}+\cos \vartheta_{1}\right)\left(L \cos \vartheta_{2}+N \sin \vartheta_{2}\right)\right]+e_{1} l_{1} \sin \vartheta_{1}\left(1+e_{2} \cos \vartheta_{2}\right)=0 \\
l_{1}\left(1+e_{2} \cos \vartheta_{2}\right)\left[\sin \vartheta_{2}\left(K \cos \vartheta_{1}+L \sin \vartheta_{1}\right)\right. \\
\left.-\left(e_{2}+\cos \vartheta_{2}\right)\left(M \cos \vartheta_{1}+N \sin \vartheta_{1}\right)\right]+e_{2} l_{2} \sin \vartheta_{2}\left(1+e_{1} \cos \vartheta_{1}\right)=0
\end{array}\right.
$$

with respect to the orbital parameters. The result of this expansion is a Taylor polynomial that describes how the stationary points move in the $\left(\vartheta_{1}, \vartheta_{2}\right)$ plane as a function of mutual orbital parameters. The algorithm described in the next section is not rigorous as the inversion of the polynomial map is not performed rigorously; i.e., the interval part of the Taylor model is not taken into account. This step is left for future work, but it is worth mentioning that, even when non rigorous computations are performed, the accuracy of the results can be suitably controlled by acting on the expansion order.

\subsection{Solution of parametric implicit equations}

In previous sections we have introduced a technique for the rigorous computation of all the solutions of the system (16). For the sake of generality, we now consider a system of $v$ implicit equations in $v$ variables with an explicit dependence on a set of $m$ parameters $\boldsymbol{p}$ (in our problem the orbital parameters), which leads to the parametric implicit equation

$$
\boldsymbol{f}(\boldsymbol{x}, \boldsymbol{p})=0 .
$$

Suppose the previous set of equations is to be solved, whose solution is represented by the function $\boldsymbol{x}(\boldsymbol{p})$ returning the value of $\boldsymbol{x}$ solving (17) for any value of the parameter $\boldsymbol{p}$. Thus, the dependence of the solution of the implicit equations on the parameters $\boldsymbol{p}$ is of interest. DA techniques can effectively handle the previous problem by identifying the function $\boldsymbol{x}(\boldsymbol{p})$ in terms of its Taylor expansion with respect to the parameter $\boldsymbol{p}$. The first step is to consider a reference value $\boldsymbol{p}^{0}$ of the parameters $\boldsymbol{p}$ and to compute the solution $\boldsymbol{x}^{0}$ of the corresponding 
implicit equation by means of a numerical method; e.g., by applying COSY-GO as seen in Sect. 6. The variable $\boldsymbol{x}$ and the parameter $\boldsymbol{p}$ are then initialized as $n$-th order DA variables; i.e.,

$$
\begin{aligned}
& {[x]=x^{0}+\delta x} \\
& {[p]=p^{0}+\delta p .}
\end{aligned}
$$

A DA-based evaluation of the function $f$ in (17) delivers the $n$-th order expansion of $f$ with respect to $\boldsymbol{x}$ and $\boldsymbol{p}$ :

$$
\delta \boldsymbol{f}=\mathcal{M}_{\boldsymbol{f}}(\delta \boldsymbol{x}, \delta \boldsymbol{p}),
$$

where $\mathcal{M}_{f}$ denotes the Taylor map for $\boldsymbol{f}$. Note that the map (19) is origin-preserving as $\boldsymbol{x}^{0}$ is the solution of the implicit equation for the nominal value of the parameter $\boldsymbol{p}^{0}$; thus, $\delta \boldsymbol{f}$ represents the deviation of $f$ from its reference value $f^{0}=0$, resulting from deviations of $\boldsymbol{x}$ and $\boldsymbol{p}$ from $\boldsymbol{x}^{0}$ and $\boldsymbol{p}^{0}$, respectively. The map (19) is then augmented by introducing the map corresponding to the identity function on $\boldsymbol{p}$ (i.e., $\delta \boldsymbol{p}=\mathcal{I}_{p}(\delta \boldsymbol{p})$ ) ending up with

$$
\left(\begin{array}{l}
\delta f \\
\delta p
\end{array}\right)=\left(\begin{array}{c}
\mathcal{M}_{f} \\
\mathcal{I}_{p}
\end{array}\right)\left(\begin{array}{l}
\delta x \\
\delta p
\end{array}\right)
$$

The $n$-th order map (20) is inverted using COSY-Infinity built-in tools, obtaining

$$
\left(\begin{array}{l}
\delta \boldsymbol{x} \\
\delta \boldsymbol{p}
\end{array}\right)=\left(\begin{array}{c}
\mathcal{M}_{\boldsymbol{f}} \\
\mathcal{I}_{\boldsymbol{p}}
\end{array}\right)^{-1}\left(\begin{array}{l}
\delta \boldsymbol{f} \\
\delta \boldsymbol{p}
\end{array}\right) .
$$

Observe that this is a full nonlinear map inversion that is obtained by applying the algorithm illustrated in Berz (1999). This algorithm reduces the map inversion problem to the solution of an equivalent fixed point problem, which can be solved with a fixed amount of effort in the DA setting.

As the goal is to compute the $n$-th order Taylor expansion of the solution manifold $\boldsymbol{x}(\boldsymbol{p})$ of (17), the map (21) is evaluated for $\delta \boldsymbol{f}=0$

$$
\left(\begin{array}{l}
\delta \boldsymbol{x} \\
\delta \boldsymbol{p}
\end{array}\right)=\left(\begin{array}{c}
\mathcal{M}_{\boldsymbol{f}} \\
\mathcal{I}_{\boldsymbol{p}}
\end{array}\right)^{-1}\left(\begin{array}{c}
0 \\
\delta \boldsymbol{p}
\end{array}\right) .
$$

The first row of map (22), which will be indicated as

$$
\delta \boldsymbol{x}=\mathcal{M}_{\left.\boldsymbol{x}\right|_{\delta=0}}(\delta \boldsymbol{p}),
$$

is the $n$-th order Taylor expansion of the solution manifold. For every value of $\boldsymbol{p}$, the approximate solution of $\boldsymbol{f}(\boldsymbol{x}, \boldsymbol{p})=0$ can be easily computed by evaluating the Taylor polynomial (23) at $\delta \boldsymbol{p}=\boldsymbol{p}-\boldsymbol{p}^{0}$. Apparently, the solution obtained by means of map (23) is a Taylor approximation of the exact solution of Eq. 17. The accuracy of the approximation depends on both the order of the Taylor expansion and the displacement $\delta \boldsymbol{p}$ from the reference value $\boldsymbol{p}^{0}$. Note that this process must be performed for any solution of the system of interest, e.g. for each stationary point of $d^{2}$ we want to analyze.

\section{Uncertain orbital parameters: Numerical experiments}

The algorithm presented in Sect. 7.1 is particularly useful to assess how the stationary points of $d^{2}$ are affected by uncertainties on the mutual orbital parameters. In this framework, we are looking for the high-order expansion of the solution of the implicit equations (16) with 
Table 9 Mutual orbital parameters uncertainty

\begin{tabular}{lllllll}
\hline$\delta r_{p_{1}}$ & $\delta e_{1}$ & $\delta r_{p_{2}}$ & $\delta e_{2}$ & $\delta i^{M}$ & $\delta w_{1}^{M}$ & $\delta w_{2}^{M}$ \\
\hline $\mathrm{AU}$ & - & $\mathrm{AU}$ & - & $\mathrm{deg}$ & $\operatorname{deg}$ & $\operatorname{deg}$ \\
\hline \pm 0.01 & \pm 0.005 & \pm 0.01 & \pm 0.005 & \pm 1 & \pm 1 & \pm 1 \\
\hline
\end{tabular}

respect to the set of uncertain parameters $\boldsymbol{p}=\left(r_{p_{1}}, e_{1}, \omega_{1}^{M}, r_{p_{2}}, e_{2}, i_{2}^{M}, \omega_{2}^{M}\right)$; i.e. we are computing

$$
\delta\left(\vartheta_{1}, \vartheta_{2}\right)=\mathcal{M}_{\vartheta \mid \nabla d^{2}=0} \delta\left(r_{p_{1}}, e_{1}, \omega_{1}^{M}, r_{p_{2}}, e_{2}, i_{2}^{M}, \omega_{2}^{M}\right)
$$

The composition of map (24) with the Taylor expansion of $d^{2}$ can be straightforwardly performed using DA tools delivering the Taylor expansion of the square distance function at the stationary point with respect to orbital parameters

$$
\delta d^{2}=\mathcal{M}_{\left.d^{2}\right|_{\nabla d^{2}=0}} \delta\left(r_{p_{1}}, e_{1}, \omega_{1}^{M}, r_{p_{2}}, e_{2}, i_{2}^{M}, \omega_{2}^{M}\right) .
$$

Thus, the evaluation of (25) delivers the distance at the stationary point MOID associated to any displaced set of mutual orbital parameters.

Maps (24) and (25) can be used to perform interesting analysis such as:

1. estimate the range of the distance function at the stationary point of interest;

2. perform fast and fully nonlinear Monte Carlo analysis by means of maps evaluation, when the statistics of the uncertain parameters are available;

3 . find the value of the parameters that minimizes the distance function within the uncertainty set.

Note that the proposed approach is based on the inversion of map (20) which is possible if and only if the linear part of this map is not singular. For the problem at hand this condition is verified if and only if the $2 \times 2$ Hessian matrix of the function $d^{2}\left(\vartheta_{1}, \vartheta_{2}\right)$ evaluated at the stationary point $\left(\vartheta_{1}^{0}, \vartheta_{2}^{0}\right)$ and the parameters set $\boldsymbol{p}^{0}$ is not singular. As a final remark, it is worth mentioning that the three proposed analyses work also for intersecting orbits, with the only inconvenient that the lower bound of the range of the square distance function will be a small negative value, due to unavoidable range overestimation.

\subsection{Range of MOID}

In this section we analyze the case in which the mutual orbital parameters are given as intervals. With reference to formalism introduced in Sect. 7.1 the set of uncertain parameters is $\boldsymbol{p}=\left(r_{p_{1}}, e_{1}, \omega_{1}^{M}, r_{p_{2}}, e_{2}, i_{2}^{M}, \omega_{2}^{M}\right)$, and we limit the study to the MOID of the four test cases of Table 1 with the range of orbital parameters reported in Table 9. Note that the uncertainty values considered are much greater then the actual ranges due to orbit determination or perturbations acting on a reasonable time window. Maps (24) and (25) are computed for each stationary point associated to the MOID of the four test cases. Once these Taylor expansions are obtained, the LDB algorithm is utilized to estimate the ranges over the given uncertainty set. The achieved results are summarized in Table 10. Note that the computation of stationary points is the most time consuming part of the algorithm, but it must be run only once. Taylor expansion, evaluation, and bounding takes fractions of second. 
Table 10 MOID Range

\begin{tabular}{llll}
\hline$\vartheta_{1}$ & $\vartheta_{2}$ & $d$ & Type \\
\hline $\operatorname{deg}$ & $\operatorname{deg}$ & $\mathrm{AU}$ & - \\
\hline$\# 1$ & {$[163.36015562,166.07394422]$} & {$[3.91941471,6.94544555]$} & {$[0.48934906,0.54966044]$} \\
$\# 2$ & {$[113.80267716,127.63861293]$} & {$[-15.48055414,-4.42603476]$} & {$[0.79792893,0.86700997]$} \\
$\# 3$ & {$[-71.91326590,-66.72145542]$} & {$[-60.55843917,-56.75299418]$} & {$[0.29821890,0.39292003]$} \\
$\# 4$ & {$[-168.54926774,-154.26478794]$} & {$[63.70715302,69.21838642]$} & {$[1.34469336,1.54060417]$} \\
\hline
\end{tabular}

\subsection{Monte Carlo simulation}

When statistics on orbital parameters are given, map (25) can be efficiently used for the nonlinear propagation of statistics. A fast and highly accurate Monte Carlo algorithm can be implemented using DA techniques based on the following steps:

1. run COSY-GO on objective function (8);

2. select the stationary point of interest (e.g. the MOID);

3. compute map (24) and (25) for the selected stationary point;

4. generate a number of samples (usually referred to as virtual asteroids) based on orbital parameters covariance matrix;

5. evaluate map (25) for all virtual asteroids;

6. perform the statistical analysis of the results.

The advantage of the proposed algorithm is that the stationary points are computed only once (i.e. a single run of COSY-GO is required). The polynomial part $P$ of the resulting Taylor models is then used to obtain maps (24) and (25), with which we study the effects of the entire range of uncertainties. As the cost of a polynomial evaluation is negligible with respect to a COSY-GO run, significant computational time saving is gained. The algorithm is applied to assess the effects of uncertain parameters $\boldsymbol{p}=\left(a, P_{1}, P_{2}, Q_{1}, Q_{2}, l\right)$ on Apophis' MOID, using the covariance matrix available at http://newton.dm.unipi.it/ neodys/epoch// 99942.eq1 to generate 10000 virtual asteroids.

Fig. 7 Statistical distribution of Apophis' MOID for 10000 virtual asteroids

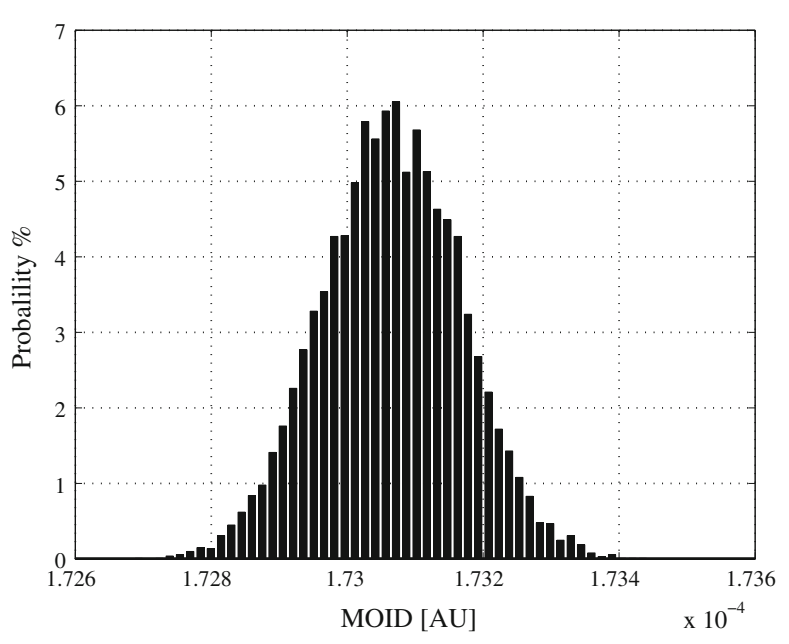


Figure 7 shows the resulting statistical distribution of Apophis' MOID, which is characterized by a mean value of $0.1730653876805878 \times 10^{-3}$ AU and a standard deviation of $1.024374440604496 \times 10^{-7}$ AU. Note that by running the algorithm of Sect. 8.1 on Apophis we obtain that, within the $3 \sigma$ values of Table 2, the MOID is confined in the range $[0.1726531026416886,0.1734719216112180] \times 10^{-3}$ AU and the corresponding stationary point lies in $\vartheta_{1} \in[99.84431986111241,99.84453465905897]$ and $\vartheta_{2} \in$ [-126.5540749528209, -126.5539424853552].

\subsection{MOID's minimum}

The last algorithm presented aims at identifying the orbital parameters that, within the given uncertainty range, minimize the MOID. This goal is achieved by running COSY-GO on an optimization problem in which maps (25) is the objective function and the orbital parameters, whose bounds are identified by their uncertainty ranges, are the optimization variables. Apophis' MOID is again used as test case: bounds are given by the $\pm 3 \sigma$ values of the equinoctial variables (see Table 2). A minimum dimension of $1 \times 10^{-6}$ is set for the variables, and an expansion order of six is considered. In $2 \times 10^{-2}$ seconds the optimizer computes the enclosure [0.172653102641443, 0.172653102641690] $\times 10^{-3}$ AU for the MOID, which corresponds to the orbital parameters $\left(a+3 \sigma_{a}, P_{1}+3 \sigma_{P_{1}}, P_{2}+3 \sigma_{P_{2}}, Q_{1}+3 \sigma_{Q_{1}}, Q_{2}-3 \sigma_{Q_{2}}\right)$.

\section{Conclusions}

A new method for the computation of all critical points of the distance function between two Keplerian orbits based on Taylor models has been presented. A global optimization problem has been formulated and solved rigorously by means of the global optimizer COSY-GO, allowing us to obtain the validated enclosure of the solutions. Some sets of orbital parameters available in the literature and asteroid Apophis have been used as test cases. Highly accurate results can be obtained efficiently in the framework of validated global optimization, allowing the method to be run for the classification of newly discovered celestial bodies. The polynomial part of the Taylor models and the tools for map inversion and composition have been exploited for the development of a set of algorithms for the management of uncertain orbital parameters. All MOID's range estimation, DA-based Monte Carlo algorithm, and MOID's minimum computations are useful tool for dealing with real problems, where the orbit determination process and/or orbital perturbations prevent the use of pointwise sets of orbital parameters. The accuracy of the analysis can be controlled by tuning the order of the Taylor expansions, enabling the application of the algorithms to real scenarios. Nevertheless, future effort will be devoted to make these algorithms fully rigorous.

Acknowledgments The authors are grateful to Cristina Parigini for her help in results visualization. The careful reading and comments of Prof. R. V. Kholshevnikov and G. F. Gronchi have improved the current version of the paper significantly.

\section{References}

Baluyev, R.V., Kholshevnikov, K.V.: Distance between two arbitrary unperturbed orbits. Celest. Mech. Dyn. Astron. 91, 287-300 (2005)

Battin, R.H.: An Introduction to the Mathematics and Methods of Astrodynamics, Second Printing. AIAA Education Series, Providence (1987) 
Berz, M., Bischof, C., Corliss, G., Griewank, A.: Computational Differentiation: Techniques, Applications, and Tools. 1-419. SIAM, Philadelphia (1996)

Berz, M.: Modern Map Methods in Particle Beam Physics. Academic Press, London (1999)

Berz, M., Makino, K., Kim, Y.: Long-term stability of the tevatron by verified global optimisation. Nucl. Instrum. Methods A558, 1-10 (2005)

Berz, M., Makino, K.: COSY INFINITY Version 9 reference manual. MSU Report MSUHEP-060803, Michigan State University, East Lansing, MI 48824, 1-84 (2006)

Dybczynski, P.A., Jopek, T.J., Seran, R.A.: On the minimum distance between two Keplerian orbits with a common focus. Celest. Mechan. 38, 345-356 (1986)

Griewank, A., Corliss, G.F.: Automatic Differentiation of Algorithms. pp. 25-31. SIAM, Philadelphia (1991)

Gronchi, G.F.: On the stationary points of the squared distance between two ellipses with a common focus. SIAM J. Sci. Comput. 24, 61-80 (2002)

Gronchi, G.F.: An algebraic method to compute the critical points of the distance function between two Keplerian orbits. Celest. Mech. Dyn. Astron. 93, 295-329 (2005)

Gronchi, G.F., Tommei, G., Milani, A.: Mutual geometry of confocal Keplerian orbits: uncertainty of the MOID and search for virtual PHAs. IAU Symposium 236, 3-14 (2007)

Hoots, F.R.: An analytical method to determine future close approaches between satellites. Celest. Mech. Dyn. Astron. 33, 143-158 (1984)

Kearfott, R.B.: Rigorous Global Search: Continuous Problems. 169-199. Kluwer Academic Publishers, Dordrecht (1996)

Kholshevnikov, K.V., Vassiliev, N.N.: On the distance function between two Keplerian elliptic orbits. Celest. Mech. Dyn. Astron. 75, 75-83 (1999)

Makino, K.: Rigorous Analysis of Nonlinear Motion in Particle Accelerators, 76-136. Ph.D. Thesis, Michigan State University, East Lansing, Michigan, USA (1998)

Makino, K., Berz, M.: Efficient control of the dependency problem based on Taylor model methods. Reliab. Comput. 5, 3-12 (1999)

Makino, K., Berz, M.: Taylor models and other validated functional inclusion methods. Int. J. Pure. Appl. Math. 6, 239-316 (2003)

Makino, K., Berz, M.: Verified global optimization with Taylor model-based range bounders. Trans. Comput. 11, 1611-1618 (2005)

Milani, A.: The asteroid identification problem I. Recovery of lost asteroids. Icarus 137, 269-292 (1999)

Sitarski, G.: Approaches of the parabolic comets to the outer planets. Acta Astronaut. 18/2, 171-195 (1968) 